\title{
Bioavailability of phenols from a phenol-enriched olive oil
}

\author{
Manuel Suárez ${ }^{1}$, Rosa M. Valls ${ }^{2}$, Maria-Paz Romero ${ }^{1}$, Alba Macià ${ }^{1}$, Sara Fernández ${ }^{2}$, Montse Giralt ${ }^{2}$, \\ Rosa Solà $^{2 *}$ and Maria-José Motilva ${ }^{1 *}$ \\ ${ }^{1}$ Food Technology Department, XaRTA-TPV, Escuela Técnica Superior de Ingeniería Agraria, University of Lleida, Av/Alcalde \\ Rovira Roure 191, 25198 Lleida, Spain \\ ${ }^{2}$ Lipid and Arteriosclerosis Research Unit (CIBERDEM), Facultat de Medicina i Ciències de la Salut, St Joan de Reus \\ University Hospital, IISPV, Universitat Rovira i Virgili, C/Sant Llorenç 21, 43201 Reus, Spain
}

(Received 15 November 2010 - Revised 21 March 2011 - Accepted 22 March 2011 - First published online 21 June 2011)

\section{Abstract}

Phenolic compounds are one of the main reasons behind the healthy properties of virgin olive oil (VOO). However, their daily intake from VOO is low compared with that obtained from other phenolic sources. Therefore, the intake of VOO enriched with its own phenolic compounds could be of interest to increase the daily dose of these beneficial compounds. To evaluate the effectiveness of enrichment on their bioavailability, the concentration of phenolic compounds and their metabolites in human plasma (0, 60, 120, 240 and 300 min) from thirteen healthy volunteers (seven men and six women, aged 25 and 69 years) was determined after the ingestion of a single dose ( $30 \mathrm{ml}$ ) of either enriched virgin olive oil (EVOO) $(961.17 \mathrm{mg} / \mathrm{kg}$ oil) or control VOO $(288.89 \mathrm{mg} / \mathrm{kg}$ oil) in a cross-over study. Compared with VOO, EVOO increased plasma concentration of the phenol metabolites, particularly hydroxytyrosol sulphate and vanillin sulphate $(P<0 \cdot 05)$. After the consumption of VOO, the maximum concentration of these peaks was reached at 60 min, while EVOO shifted this maximum to $120 \mathrm{~min}$. Despite these differences, the wide variability of results indicates that the absorption and metabolism of olive oil phenols are highly dependent on the individual.

Key words: Bioavailability: Human plasma: Phenol-enriched olive oil: Plasma phenol metabolites

Over the last decade, epidemiological studies have proven that a Mediterranean diet, rich in vegetables, fruit and legumes, is correlated with a low incidence of CHD and cancer $^{(1)}$. Within this nutritional pattern, virgin olive oil (VOO) plays an important role, being the main source of fat in the diet in this area. The beneficial effects of VOO were initially attributed to its fatty acid composition, rich in MUFA. Nevertheless, further studies have highlighted the importance of its phenolic composition, especially after the publication of the results of the EUROLIVE project, which demonstrated that the increase in the HDL-cholesterol level and the decrease in the lipid oxidation damage after VOO consumption were highly correlated with its phenolic content ${ }^{(2,3)}$. Thus, phenolic compounds from VOO (mainly phenolic alcohols, secoiridoid derivatives, phenolic acids, lignans and flavonoids) have been reported to have anti-oxidant ${ }^{(4)}$, anti-inflammatory ${ }^{(5)}$, anti-atherogenic $^{(6)}$ and anti-carcinogenic properties ${ }^{(7)}$.

The metabolic fate of phenolic compounds after ingestion has been the subject of study by the scientific community to find out the mechanisms through which they exert their activity into the organism. It is known that polyphenols suffer strong metabolism phase I and phase II, in which they are hydrolysed (phase I) and later conjugated (phase II) into their glucuronidated, methylated and sulphated forms in order to be absorbed ${ }^{(8)}$. In the case of VOO, studies have focused on some specific compounds such as hydroxytyrosol and tyrosol as they have been reported as the most biologically active. Thus, Visioli et $a l^{(9)}$ were the first researchers who identified these compounds in urine, both in the conjugated and in their free forms. In addition, this study showed that hydroxytyrosol and tyrosol are dosedependently absorbed in humans. Later, the same research group reported the presence of homovanillic alcohol in urine, a compound that is derived from hydroxytyrosol by the action of the enzyme catechol-O-methyltransferase ${ }^{(10)}$. On the other hand, the study by Miró-Casas et al. ${ }^{(11)}$ reported the presence of conjugated forms of hydroxytyrosol in plasma. The importance of these compounds has been reinforced by the demonstration that they can aid in preventing the oxidation of $\mathrm{LDL}^{(12)}$. Recently, an association between

Abbreviations: 3,4-DHPEA-EDA, dialdehydic form of elenolic acid linked to hydroxytyrosol; EVOO, enriched virgin olive oil; MOPET, 3-methoxy-4hydroxy-phenylethanol; UPLC, ultra performance liquid chromatography; VOO, virgin olive oil.

*Corresponding authors: Dr M.-J. Motilva, fax + 34973 702596, email motilva@tecal.udl.es; R. Sola, fax +34 977759322, email rosa.sola@urv.cat 
hydroxytyrosol and LDL in plasma has been described, a fact that could have important physiological implications ${ }^{(13)}$. All these studies demonstrated the importance of hydroxytyrosol and tyrosol; however, little has been done about the other components of the phenolic fraction of VOO, which have also been shown to have high biological activity such as the dialdehydic form of elenolic acid linked to hydroxytyrosol $(3,4-D H P E A-E D A)^{(14)}$.

More recent studies have indicated that the beneficial effects of the phenolic compounds from VOO are not only due to their anti-oxidant and related activities, but they also have a nutrigenomic effect, which means that their intake has a direct impact on the human genome. Thus, a study carried out by Konstantinidou et $a l^{(15)}$ showed that the phenols from VOO have a significant role in the down-regulation of proatherogenic genes such as IFN- $\gamma, A R H G A P 15$ and $I L 7 R$. This reinforces the importance of including VOO in the regular diet (the Food and Drug Administration recommends a daily intake of $23 \mathrm{~g}$ of olive oil).

Nevertheless, the amount of phenolic metabolites from VOO detected in human plasma samples is highly variable and depends on both extraction and analytical procedure. Thus, Vissers et $a l^{(16)}$ reported that the bioavailability of these compounds in ileostomed volunteers was up to $66 \%$ of the total ingested. Considering a mean intake of $50 \mathrm{~g}$ of $\mathrm{VOO} / \mathrm{d}$, these authors estimated that the total amount of phenolic compounds in the plasma samples could be at most $0.06 \mu \mathrm{mol}$ of hydroxytyrosol equivalents/1 plasma. However, some studies revealed that a higher concentration is required to protect LDL from oxidation. For example, Leenen et al. ${ }^{(17)}$ concluded that at least $50-100 \mu \mathrm{mol}$ of phenolic compounds from VOO/1 plasma are required to have a protective effect on LDL. Therefore, according to these results, enough phenols are unlikely to be obtained from VOO to have a direct protective effect on LDL. In addition, the concentration of phenolic compounds in commercial VOO is widely dependent on agronomical and technical factors ${ }^{(18)}$. This decreases the ingestion of phenols from VOO compared with the doses ingested from other sources and makes it difficult to ensure a regular dose of phenols through the daily intake of VOO. For this reason, the enrichment of VOO with their own phenols, thus giving a high and standardised content of phenolic compounds, could be desirable in order to increase the daily intake of phenols without the drawback of a higher energy content.

The aim of the present study was to evaluate the effect of the enrichment of VOO with their own phenolic compounds in their bioavailability in human subjects. To fulfil this objective, the phenol metabolites were identified and quantified in human plasma samples obtained after an acute ingestion of $30 \mathrm{ml}$ of a phenol-enriched VOO (EVOO). Plasma samples were obtained at 0,60,120, 240 and $300 \mathrm{~min}$ in order to study the pharmacokinetic behaviour of these metabolites. The results were compared with those obtained after the ingestion of the VOO (without phenol addition).

\section{Experimental methods}

\section{Chemicals and reagents}

Standard phenolic compounds were used to obtain calibration curves to quantify the compounds in the olive oils and in the plasma samples. Apigenin, luteolin, hydroxytyrosol, tyrosol and $p$-coumaric acid were purchased from Extrasynthese (Genay, France). Caffeic and homovanillic acids were purchased from Fluka Company (Buchs, Switzerland). (+)-Pinoresinol was acquired from Arbo Nova (Turku, Finland), and catechol was from Sigma-Aldrich (Schnelldorf, Germany). The secoiridoid derivatives 3,4-DHPEA-EDA, the dialdehydic form of elenolic acid linked to tyrosol and the lignan acetoxypinoresinol are not available commercially, and were isolated from virgin olive by semi-preparative HPLC, as described by Artajo et $a l .{ }^{(19)}$. A stock solution of each standard compound was dissolved in methanol, and all the solutions were stored in a dark flask at $-40^{\circ} \mathrm{C}$.

Methanol (HPLC grade), acetonitrile (HPLC grade) and acetic acid were provided by Scharlau Chemie (Barcelona, Spain). Ortho-phosphoric acid (85\%) was purchased from Panreac (Barcelona, Spain). Water was MilliQ quality (Millipore Corporation, Bedford, MA, USA).

\section{Virgin olive oil}

The VOO used as control and as matrix for phenolic enrichment was from the Siurana Protected Denomination of Origin (Catalonia, Spain), which is produced exclusively from the Arbequina cultivar. On the other hand, the samples of the olive cake used to obtain the phenolic extract for phenol enrichment were taken from a commercial olive oil mill from the olive-growing area of Les Garrigues (Catalonia, Spain), which works by the two-phase centrifugation system. These samples were taken at the decanter outlet, and liquid $\mathrm{N}_{2}$ was immediately added to avoid oxidative damage. The samples were stored at $-40^{\circ} \mathrm{C}$ until the extraction of the phenolic compounds.

\section{Preparation of the phenol-enriched olive oil}

The EVOO was prepared by the addition of an extract rich in the main phenolic compounds of VOO. This extract was obtained from the olive cake following the method described by Suárez et $a l .{ }^{(20)}$ and basically included secoiridoid derivatives (89.4\%), phenyl alcohols (3.5\%) and flavonoids (6.0\%).

To carry out phenolic enrichment of the oil, $7 \mathrm{mg}$ of olive cake phenolic extract $/ \mathrm{ml}$ oil and $0.3 \%(\mathrm{p} / \mathrm{v})$ of lecithin (Emulpur; Cargill, Barcelona, Spain) were dissolved in ethanol-water (50:50, v/v) and added to the oil using a Polytron (Kinematica, Littau, Switzerland) until completely homogenised. Finally, the EVOO was filtered through a paper filter Ahlstrom (Ahlstrom S.A., Barcelona, Spain) and bottled in dark bottles. The VOO used as control (without added phenolic compounds) was also submitted to the process of mixing and filtering to ensure equal conditions in all the olive oils under study. The phenolic compounds of both the VOO and the EVOO were extracted and characterised with the method described in our previous study ${ }^{(21)}$. 


\section{Volunteers and experimental design}

The present study was conducted according to the guidelines laid down in the Declaration of Helsinki, and all procedures involving human subjects were approved by the Ethics Committee of Clinical Research of Sant Joan University Hospital, Reus, Spain (Trial Registration clinicaltrials.gov Identifier: ISRCTN03450153). The subjects gave written informed consent. A total of sixteen healthy volunteers (eight women and eight men) were recruited to carry out the study. However, at the moment of ingestion, three of the volunteers (two women and one man) gave up the study, thus reducing the final number of volunteers to thirteen. The volunteers, aged between 25 and 69 years, were considered healthy according to the results obtained after physical examination and routine laboratory tests. The men had a mean weight of 78.3 (SD $13.0) \mathrm{kg}$ and the women had a mean weight of 64.5 (SD 5.7) kg, while the BMI was 26.7 (SD 1.9) $\mathrm{kg} / \mathrm{m}^{2}$ for men and $24.9(\mathrm{SD} 1.5) \mathrm{kg} / \mathrm{m}^{2}$ for women.

A randomised, controlled, cross-over trial was designed using VOO (without phenol addition) and EVOO. Thus, the volunteers who consumed VOO in the first intervention ingested the EVOO in the second intervention after a 2-week washout period (Fig. 1) and vice versa, volunteers who consumed the EVOO in the first intervention ingested the VOO in the second intervention. The participants were instructed to follow a stabilisation diet with $10 \%$ of SFA during the week before the postprandial test and the day before a polyphenol free diet was prescribed. Between the tests, the volunteers followed the stabilisation diet. The recommendations were to avoid VOO, olives, fresh fruits or juices, wine, chocolate, coffee, tea, soya, legumes and beer. The participants were instructed to perform intense physical exercise $3 \mathrm{~d}$ before the postprandial test.

Acute ingestion of the oil was done at 08.00 hours after an overnight fast. The VOO was included in the study to enable the comparison of the resulting data and to extract conclusions.

\section{Plasma samples}

Plasma samples were obtained by venepuncture from volunteers after the ingestion of $30 \mathrm{ml}$ of either VOO or EVOO. The blood samples were collected under basal conditions

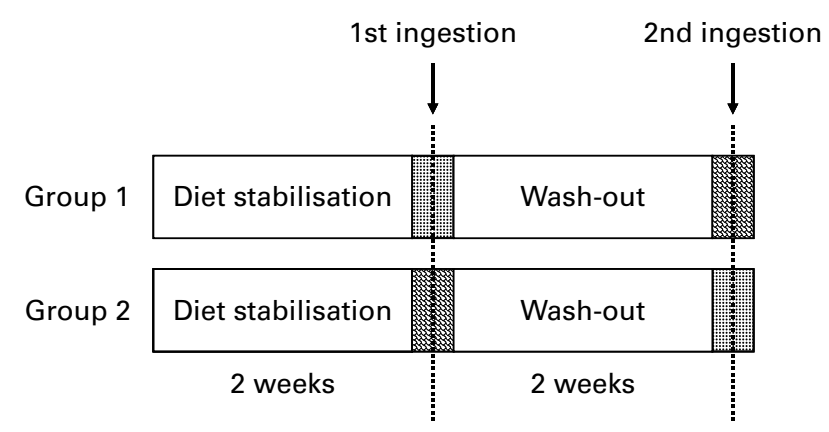

Fig. 1. Study design of the acute ingestion study of control and phenolenriched olive oil. 圈, $30 \mathrm{ml}$ of control olive oil; 图, $30 \mathrm{ml}$ of phenol-enriched olive oil. (after a $12 \mathrm{~h}$ overnight fasting period) and 60, 120, 240 and $300 \mathrm{~min}$ after the consumption of $30 \mathrm{ml}$ of either enriched or control VOO. To obtain plasma samples at every measuring point, blood $(50 \mathrm{ml})$ was collected into Vacutainer ${ }^{\mathrm{TM}}$ tubes containing EDTA as an anti-coagulant. They were protected from light with $\mathrm{Al}$ foil and centrifuged for $15 \mathrm{~min}$ at $1500 \mathrm{~g}$ and $4^{\circ} \mathrm{C}$ (H-103RS; Kokusan, Tokyo, Japan). After that, the plasma was immediately separated from the cells and kept at $-80^{\circ} \mathrm{C}$ until analysis.

The extraction of the phenolic compounds from the plasma samples was carried out following the method developed and validated in our previous study ${ }^{(22)}$ using microelution plates (Waters, Milford, MA, USA) packed with $2 \mathrm{mg}$ of OASIS HLB sorbent (Waters).

\section{Analysis of phenolic compounds in olive oils and plasma samples}

The analysis of the phenolic compounds from both oils (VOO and EVOO) and plasma samples was carried out by ultra performance liquid chromatography (UPLC) coupled to tandem MS. The UPLC system consisted of an AcQuity ${ }^{\text {TM }}$ UPLC equipped with a Waters binary pump system using an AcQuity UPLC $^{\mathrm{TM}}$ BEH $\mathrm{C}_{18}$ column $(1.7 \mu \mathrm{m}, 100 \mathrm{~mm} \times 2.1 \mathrm{~mm}$ inner diameter). During the analysis, the column was kept at $30^{\circ} \mathrm{C}$ and the flow rate was $0.4 \mathrm{ml} / \mathrm{min}$, using MilliQ water-acetic acid (100:0.2, v/v) as solvent $\mathrm{A}$ and acetonitrile as solvent $\mathrm{B}$. The elution gradient was the one reported in our previous study $^{(21)}$.

Full-scan mode MS and MS/MS, based on neutral loss scan and product ion scan, were used to identify and quantify the phenolic compounds and their metabolites from plasma samples. These techniques are very effective in verifying the structural information of the compounds when no commercially standards are available. To start with, the analyses were carried out in full-scan mode (from 80 to $800 \mathrm{~m} / z$ ) by applying different cone voltages from 20 to $60 \mathrm{~V}$. The MS spectrum obtained when low cone voltages were used gave information about the precursor ion or the $[\mathrm{M}-\mathrm{H}]^{-}$. On the other hand, when high cone voltages were applied, specific fragment ions were generated and the MS spectrum gave information about their structure. The structural information was also verified by using the product ion scan and neutral loss scan in the MS/MS mode. In the product ion scan experiments, the product ions are produced by collision-activated dissociation of the selected precursor ion in the collision cell. Neutral loss scans of 80 and 176 units were used to characterise the sulphate and glucuronide forms, respectively. The detection and quantification of the phenolic compounds and their metabolites were then performed based on their ion fragmentation in the MS/MS mode using selected reaction monitoring (SRM) and double SRM.

Due to the lack of standards for these metabolites, they were quantified with the calibration curves corresponding to their phenolic precursors. Hydroxytyrosol and tyrosol metabolites were quantified using the calibration curves of hydroxytyrosol and tyrosol, respectively; homovanillic acid, vanillic acid and vanillin metabolites were quantified using the 
calibration curve of homovanillic acid; $p$-coumaric acid, $p$-hydroxybenzoic acid and ferulic acid metabolites were quantified by means of the $p$-coumaric acid calibration curve. Apigenin metabolites were quantified with the apigenin calibration curve.

\section{Statistical analysis and pharmacokinetic parameters}

Values are presented as means and standard deviations. Data analysis was performed using Statgraphics plus v.5.1 software (Manugistics, Inc., Rockville, MA, USA). The normality of the data was evaluated by means of the values of the coefficients of asymmetry and kurtosis. Significant differences among the results from the two olive oils were evaluated by the Kruskal-Wallis ANOVA $(P<0.05)$.

On the other hand, kinetic parameters of the different metabolites of the phenolic compounds were calculated by means of pharmacokinetic (PK) functions (for Microsoft Excel). This software allowed us to determine the maximum concentration of the metabolites $\left(C_{\max }\right)$ and their area under the curve, which can be used to compare the effect of the ingestion of the different oils (control or phenol-enriched).

\section{Results and discussion}

\section{Characterisation of the virgin olive oils}

The phenolic profiles of both VOO and EVOO were analysed by UPLC-electrospray ionisation-MS/MS. As can be seen in Table 1 , the addition of the olive cake extract significantly

Table 1. Phenolic composition of the control and phenol-enriched olive oil determined by ultra performance liquid chromatography (UPLC)electrospray ionisation (ESI)-MS/MS

(Mean values and standard deviations)

\begin{tabular}{|c|c|c|c|c|}
\hline \multirow[b]{2}{*}{ Phenolic compounds } & \multicolumn{2}{|c|}{$\begin{array}{l}\text { Control virgin } \\
\text { olive oil } \\
\text { (mg/kg oil) }\end{array}$} & \multicolumn{2}{|c|}{$\begin{array}{c}\text { Phenol-enriched } \\
\text { olive oil } \\
\text { (mg/kg oil) }\end{array}$} \\
\hline & Mean & SD & Mean & SD \\
\hline Hydroxytyrosol & 0.37 & 0.06 & $6 \cdot 64$ & 0.64 \\
\hline Tyrosol & 1.03 & 0.09 & $8 \cdot 70$ & 2.00 \\
\hline Total phenyl alcohols & \multicolumn{2}{|c|}{1.40} & \multicolumn{2}{|c|}{$15 \cdot 3$} \\
\hline Vanillic acid & 0.37 & 0.02 & 3.94 & 0.18 \\
\hline$p$-Coumaric acid & 0.08 & 0.02 & 0.84 & $0 \cdot 11$ \\
\hline Vanillin & $0 \cdot 16$ & 0.01 & 1.44 & 0.15 \\
\hline Total phenolic acids & \multicolumn{2}{|c|}{0.61} & \multicolumn{2}{|c|}{$6 \cdot 21$} \\
\hline 3,4-DHPEA-EDA & $45 \cdot 4$ & $9 \cdot 29$ & $527 \cdot 8$ & 30.55 \\
\hline 3,4-DHPEA-EA & $15 \cdot 1$ & 0.97 & $26 \cdot 2$ & 5.58 \\
\hline 3,4-DHPEA-AC & $52 \cdot 8$ & $11 \cdot 28$ & 102.5 & $18 \cdot 26$ \\
\hline$p$-HPEA-EDA & $9 \cdot 72$ & 1.03 & $24 \cdot 1$ & $4 \cdot 14$ \\
\hline Total secoiridoid derivatives & \multicolumn{2}{|c|}{$123 \cdot 1$} & \multicolumn{2}{|c|}{680.6} \\
\hline Pinoresinol & $115 \cdot 8$ & $10 \cdot 19$ & $173 \cdot 1$ & $14 \cdot 18$ \\
\hline Acetoxypinoresinol & $46 \cdot 3$ & 5.91 & $78 \cdot 8$ & 9.39 \\
\hline Total lignans & \multicolumn{2}{|c|}{$162 \cdot 1$} & \multicolumn{2}{|c|}{251.9} \\
\hline Luteolin & 1.44 & 0.26 & $6 \cdot 28$ & 0.25 \\
\hline Apigenin & 0.27 & 0.01 & 0.80 & 0.04 \\
\hline Total flavonoids & \multicolumn{2}{|c|}{1.71} & \multicolumn{2}{|c|}{7.08} \\
\hline Total phenolic compounds & \multicolumn{2}{|c|}{288.9} & \multicolumn{2}{|c|}{$961 \cdot 2$} \\
\hline
\end{tabular}

3,4-DHPEA-EDA, dialdehydic form of elenolic acid linked to hydroxytyrosol; 3,4DHPEA-EA, oleuropein aglycone; 3,4-DHPEA-AC, 4-(acetoxyethyl)-1,2-dihidroxybenzene; $p$-HPEA-EDA, dialdehydic form of elenolic acid linked to tyrosol. increased the concentration of all the phenolic compounds in the oils. The $10 \cdot 95$-fold increase experimented by the phenyl alcohol fraction, made up of hydroxytyrosol and tyrosol, was especially remarkable. These compounds are biologically active, so it is very interesting to have them at a higher and optimal concentration in the olive oil available to be absorbed by the organism after ingestion.

It is also important to highlight the increase observed in the secoiridoid derivatives and the flavonoid group. The importance of the secoiridoid derivatives is based on their chemical structure. These compounds are precursors of phenyl alcohols, which, as was stated earlier, are very active compounds. In addition, recent studies have demonstrated that some secoiridoid derivatives, such as 3,4-DHPEA-EDA, are even better at protecting erythrocytes from oxidative damages than hydroxytyrosol ${ }^{(23)}$. Therefore, the secoiridoid derivatives are valuable compounds that could have a beneficial effect on the organism. On the other hand, flavonoids are the most widespread natural phenolic compounds whose important activity is due to their characteristic triple aromatic ring chemical structure. However, their concentration in VOO is very low compared with that obtained from other phenolic sources. Thus increasing the amount of flavonoids in olive oils is of interest to increase their intake from this source.

Therefore, the greater concentration of phenolic compounds in the EVOO is expected to increase the level of their corresponding metabolites in human plasma after the consumption of the oils due to the higher amount available to be absorbed by the organism.

\section{Phenol metabolite identification and quantification in human plasma}

Phenolic compounds and their corresponding metabolites in the plasma samples after ingestion of either VOO or EVOO were identified and quantified following the procedure described by Suárez et al. ${ }^{(22)}$. Table 2 shows the main chromatographic peaks that appeared in the chromatograms. On the basis of their retention time, corresponding molecular weight and ion transition, and the information obtained from the literature, we proposed which phenolic compounds and metabolites could match these peaks. Some of the compounds had more than one chromatographic peak, appearing at different retention times but maintaining the molecular weight and the mass spectrometric fragments. This suggests the existence of several isomerical forms of these compounds in the plasma samples.

As expected, most of the metabolites identified in the plasma were derived from the secoiridoids and phenyl alcohol groups and appeared in their conjugated form, especially sulphated and glucuronidated. This could suggest that basically the phenolic compounds from olive oil follow two different pathways: one mediated by the action of the sulphotransferase enzymes and the other governed by the glucurotransferases. This agrees with the results obtained by other authors, namely De la Torre-Carbot et al. ${ }^{(24)}$ who identified hydroxytyrosol and tyrosol in their glucuronidated and sulphated forms in human LDL after the intake of 
Table 2. Chromatographic parameters and proposed compound of the main chromatographic peaks observed in the plasma samples after ingestion of the olive oils (control and phenol-enriched)

\begin{tabular}{llccl}
\hline Peak & Retention time $(\mathrm{min})$ & {$[\mathrm{M}-\mathrm{H}]^{-}$} & Fragments MS/MS & Proposed compound \\
\hline 1 & $3 \cdot 62 ; 10 \cdot 65$ & 137 & 93 & Hydroxybenzoic acid \\
2 & $4 \cdot 63,6 \cdot 52$ & 151 & 107 & Hydroxyphenylacetic acid \\
3 & $6 \cdot 55$ & 151 & 136 & Vanillin \\
4 & $4 \cdot 85$ & 167 & 123 & Vanillic acid \\
5 & $5 \cdot 17$ & 179 & 135 & Caffeic acid \\
6 & $17 \cdot 1$ & 181 & 137 & Homovanillic acid derivative \\
7 & $10 \cdot 14$ & 197 & 153 & 3,4-DHPEA-EA derivative \\
8 & $8 \cdot 55 ; 19 \cdot 15$ & 217 & 137 & Tyrosol sulphate \\
9 & $5 \cdot 06 ; 7 \cdot 98 ; 12 \cdot 45$ & 230 & 150 & 4-Methoxy-phenylethanol sulphate \\
10 & $9 \cdot 32 ; 14 \cdot 11$ & 231 & 151,136 & Vanillin sulphate \\
11 & $6 \cdot 15$ & 233 & 153,123 & Hydroxytyrosol sulphate \\
12 & $6 \cdot 67 ; 13 \cdot 5 ; 21 \cdot 75$ & 243 & 163,119 & Coumaric acid sulphate \\
13 & $11 \cdot 77 ; 16 \cdot 86 ; 19 \cdot 3$ & 245 & 165 & 4-Methoxy-phenylacetic acid sulphate \\
14 & $10 \cdot 05$ & 247 & 167,123 & Vanillic acid sulphate \\
15 & $19 \cdot 15 ; 23 \cdot 83$ & 259 & 179,135 & Caffeic acid sulphate \\
16 & $9 \cdot 48$ & 261 & 181 & Homovanillic acid sulphate \\
17 & $11 \cdot 27$ & 273 & 193,134 & Ferulic acid sulphate \\
18 & $4 \cdot 56 ; 6 \cdot 74$ & 327 & 150 & 4-Methoxy-phenylethanol glucuronide \\
19 & $8 \cdot 77 ; 18 \cdot 97$ & 327 & 151,136 & Vanillin glucuronide \\
20 & $7 \cdot 00$ & 369 & 193,134 & Ferulic acid glucuronide \\
21 & 10.57 & 373 & 197 & 3,4-DHPEA-EA derivative glucuronide \\
22 & $15 \cdot 15$ & 445 & 269 & Apigenin glucuronide \\
23 & $10 \cdot 69 ; 13 \cdot 24$ & 495 & 319,153 & 3,4-DHPEA-EDA glucuronide \\
\hline
\end{tabular}

3,4-DHPEA-EA, oleuropein aglycone; 3,4-DHPEA-EDA, dialdehydic form of elenolic acid linked to hydroxytyrosol.

VOO. Thus, these conjugated forms are good biomarkers of phenolic compounds of VOO.

The chromatographic analysis of the plasma samples also revealed the existence of other compounds (either conjugated or in their free form), including phenolic acids (hydroxybenzoic, hydroxyphenylacetic, ferulic and caffeic acids and their derivatives) and flavonoids (apigenin glucuronide).

With regard to the metabolites derived from hydroxytyrosol, the appearance of compounds structurally related to homovanillic acid suggests the existence of a methylation process in the organism, probably due to the activity of the enzyme catechol-O-methyltransferase. D'Angelo et al. ${ }^{(25)}$ proposed a metabolic pathway for hydroxytyrosol in which the action of this enzyme promoted the appearance of homovanillic acid and its conjugated forms. In the case of the plasma samples obtained after the ingestion of both VOO and EVOO, we were able to identify the metabolites that appear at the end of this chain of reactions: hydroxytyrosol sulphate, homovanillic acid derivative and homovanillic acid sulphate. Homovanillic acid derivative was a compound that presented the same precursor and product ions as homovanillic acid but eluted at a different retention time from the commercial standard. The non-identification of free hydroxytyrosol and the other intermediate forms may suggest that these compounds are rapidly subjected to the activity of the enzymes, originating in the sulphated or methyl-sulphated forms, which are more likely to be absorbed by the organism. Fig. 2 shows the metabolic pathway of hydroxytyrosol proposed by D'Angelo et al. ${ }^{(25)}$ with some variations, indicating in bold the metabolites identified in the plasma samples after the ingestion of olive oil.

Similar to the metabolic pathway described in hydroxytyrosol, the analysis of the plasma samples revealed the existence of some methylated metabolites that probably originated from tyrosol. Thus, peaks 9, 13 and 18 given in Table 2 could be formed from tyrosol, with an analogous chemical structure to the one obtained as a result of the activity of catechol-Omethyltransferase on hydroxytyrosol. Peak 9 is equivalent to 3-methoxy-4-hydroxy-phenylethanol (MOPET) sulphate, peak 13 is equivalent to the homovanillic sulphate and peak 18 was the result of the glucuronidation of the analogue structure of MOPET in tyrosol.

These results contrast with those obtained by GarcíaVillalba et al. ${ }^{(26)}$ who did not identify methyl conjugate metabolites from tyrosol in urine samples after the ingestion of $50 \mathrm{ml}$ of VOO. They attributed this to the lack of activity of catechol-O-methyltransferase on this compound due to the need for an ortho-diphenolic structure of this enzyme. The appearance of the methylated compounds in the plasma samples in the present study could suggest the presence of enzymatic activity able to methylate tyrosol. Thus, Fig. 3 shows the proposed metabolic pathways that tyrosol could undergo in the organism, highlighting in bold the metabolites identified in the plasma samples after the consumption of the VOO. Based on the pathway described by D'Angelo et al. ${ }^{(25)}$, we hypothesised that tyrosol could also be affected by the activity of alcohol dehydrogenase and aldehyde reductase, although the metabolites produced by the activity of these enzymes were not found. This could be due to the high instability of these compounds and the possible preference for methylation.

\section{Pharmacokinetic parameters of phenol metabolites}

The concentration of the phenolic compounds and their metabolites in the plasma samples was monitored 0, 60, 120, 


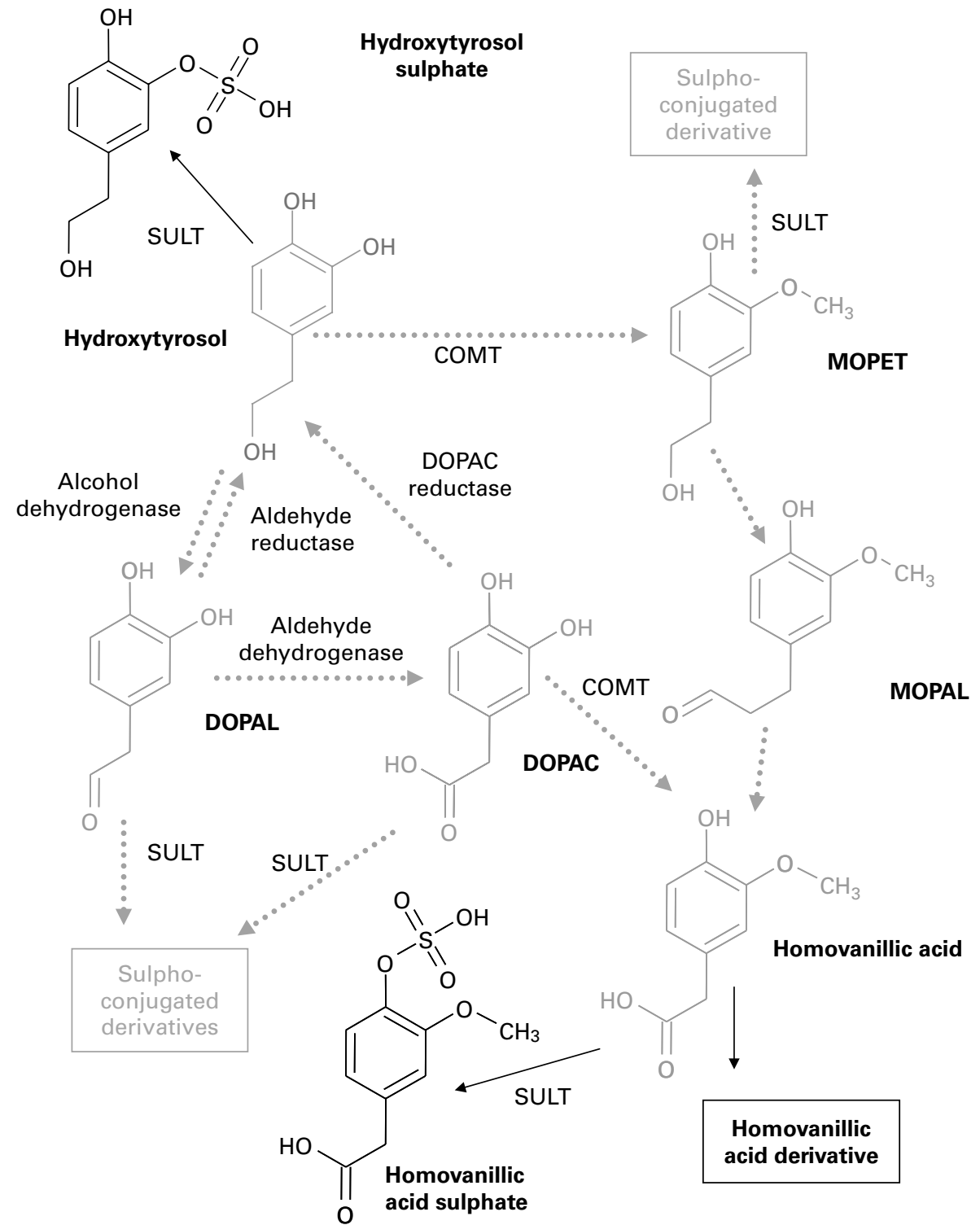

Fig. 2. Metabolic pathways of hydroxytyrosol (3,4-dihydroxy-phenylethanol; adapted from D'Angelo et al. $\left.{ }^{(25)}\right)$. The chemical structures in bold were identified in the plasma samples after olive oil ingestion. MOPET, 3-methoxy-4-hydroxy-phenylethanol; SULT, sulphotransferase; COMT, catechol-O-methyltransferase; DOPAL, 3,4-dihydroxy-phenylacetaldehyde; DOPAC, 3,4-dihydroxy-phenylacetic acid; MOPAL, 3-methoxy-4-hydroxy-phenylacetaldehyde; homovanillic acid, 4-hydroxy-3methoxy-phenylacetic acid.

240 and 300 min after the ingestion of either VOO or EVOO, and the difference between their bioavailability depending on the olive oil consumed was evaluated.

In general, the data revealed that the amount of phenolic metabolites in the plasma samples obtained after the ingestion of the EVOO was higher than that obtained after the intake of the VOO. Tables 3 and 4 show the pharmacokinetic parameters obtained after analysing the chromatographic results with the PK functions software. This software allows us to tentatively determine the value of the maximum concentration of the compounds in the plasma $\left(C_{\max }\right)$ and the area under the curve in the interval of study.

As can be seen in Table 3, almost all the identified compounds that are involved in the hydroxytyrosol pathway reached higher concentrations in plasma samples obtained after the ingestion of EVOO compared with the values obtained after the intake of VOO. This suggests that, effectively, the enrichment of the olive oil with the phenol extract successfully produced an increase in the concentration of these compounds in plasma after oil consumption. This is desirable as a way to achieve a concentration of phenol metabolites in plasma able to produce a real effect in the organism. However, the variability of the results among the different volunteers was very high, as can be observed in the area under the curve range. This is because bioavailability of phenolic compounds not only depends on their concentration in the oil, but is also affected by each individual's characteristic parameters, such as their enzymatic activity and habitual diet. Thus, despite the mean values being higher after the consuming EVOO, only the concentration of hydroxytyrosol sulphate and vanillin sulphate 


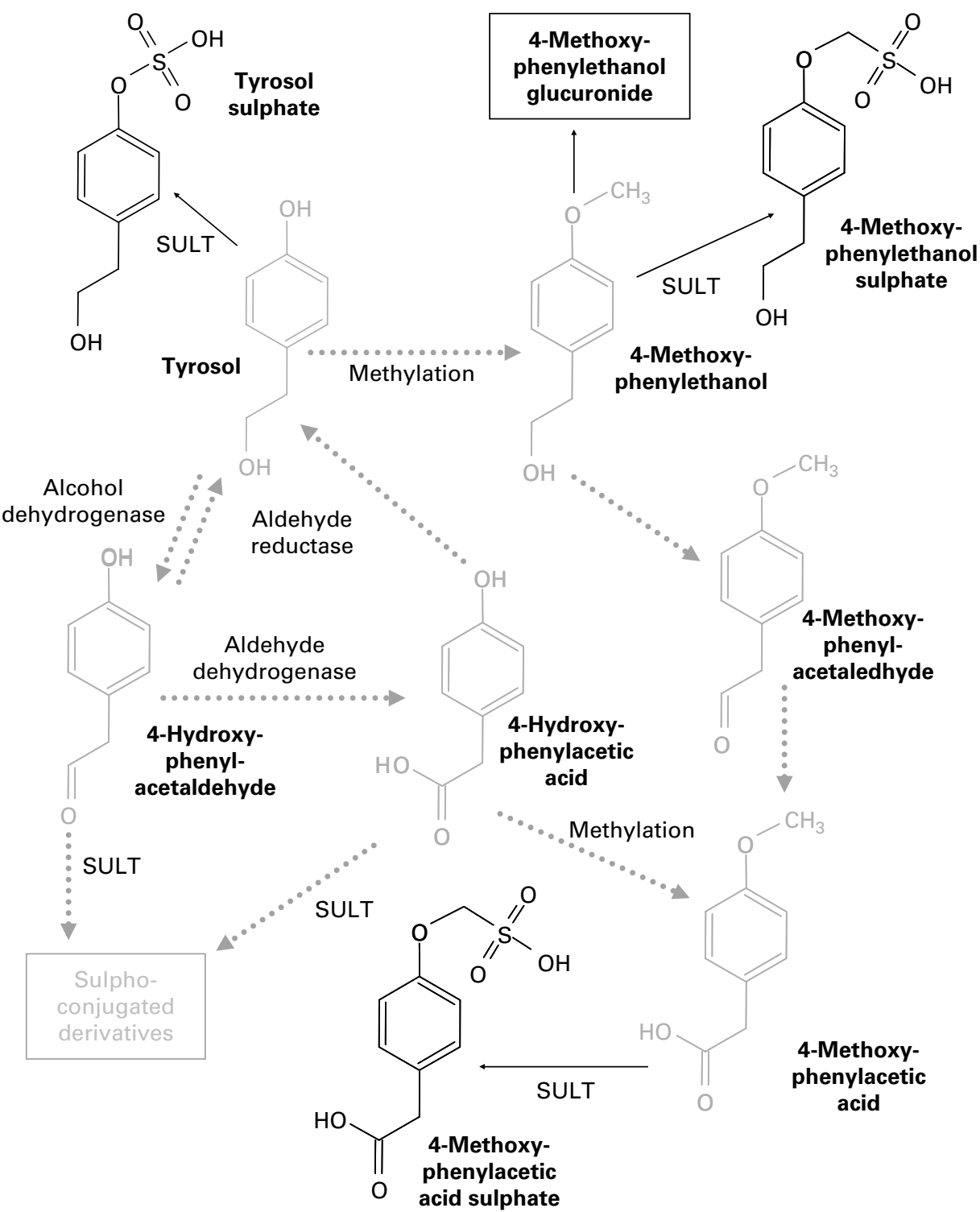

Fig. 3. Proposed metabolic pathways of tyrosol. The chemical structures in bold were identified in the plasma samples after olive oil ingestion. SULT, sulphotransferase.

was statistically higher than in the VOO. This inter-individual variability was also reported by other authors. D'Archivio et $a{ }^{(27)}$ pointed out in their review about the bioavailability of phenolic compounds in human subjects, suggesting that one important factor to be considered in these studies is the different genomic profile of the individuals. This influence of the genomic profile in the absorption and effectiveness of the polyphenols suggests that in the future further studies should be carried out to evaluate the suitability of the introduction of a personalised factor in the design of functional foods.

On the other hand, although in all the cases an increase in the concentration of the peaks for the tyrosol metabolites was detected after consumption of both VOO and EVOO, no significant differences between these oils were observed. In addition, the mean values were very similar, suggesting that the bioavailability of these compounds was not affected by phenol enrichment. It is possible that complete absorption of the phenols ingested with EVOO could be limited by the membrane carriers. However, the intestinal mechanism of the gastrointestinal absorption of polyphenols remains poorly understood, and the membrane carriers that could be involved in polyphenol absorption have not been identified. The major components of the VOO phenolic fraction, the secoiridoid derivatives (oleuropein and ligstroside aglycones), are hydrolysed in the gastrointestinal tract ${ }^{(28)}$, after which the transport of the resulting polar phenols, tyrosol and hydroxytyrosol, might occur via passive diffusion. In a previous study, we have shown that there was extensive transport of tyrosol and hydroxytyrosol and their conjugates to the basolateral side using a Caco-2/TC7 model of the small intestine ${ }^{(29)}$. In general, apical loading of individual phenols resulted in time-dependent efflux of different conjugates. However, future studies are necessary to establish the optimal dose:bioavailability ratio. 
Table 3. Pharmacokinetic parameters of the metabolites derived from the secoiridoids in plasma samples after the intake of either control or phenolenriched olive oil $(n 13)$

(Mean values, standard deviations and ranges)

\begin{tabular}{|c|c|c|c|c|c|c|c|}
\hline \multirow[b]{2}{*}{ Phenolic compound } & \multirow[b]{2}{*}{ Oil } & \multicolumn{2}{|c|}{$C_{\max }(\mu \mathrm{mol} / \mathrm{l})$} & \multicolumn{2}{|c|}{$\begin{array}{c}\text { AUC } \\
(\mu \mathrm{mol} \times \min / l)\end{array}$} & \multirow{2}{*}{$\begin{array}{c}\text { AUC range } \\
(\mu \mathrm{mol} \times \mathrm{min} / \mathrm{l})\end{array}$} & \multirow[b]{2}{*}{$P$} \\
\hline & & Mean & SD & Mean & SD & & \\
\hline \multirow[t]{2}{*}{ Hydroxytyrosol sulphate } & Control & 0.53 & $0 \cdot 30$ & 103 & 65 & $50-238$ & \multirow{2}{*}{$<0.05$} \\
\hline & Enriched & 0.86 & 0.24 & 159 & 49 & $93-287$ & \\
\hline \multirow[t]{2}{*}{ Homovanillic acid derivative } & Control & 0.53 & 0.29 & 132 & 59 & $80-246$ & \multirow{2}{*}{ NS } \\
\hline & Enriched & 0.34 & $0 \cdot 10$ & 80 & 20 & $63-102$ & \\
\hline \multirow[t]{2}{*}{ Homovanillic acid sulphate } & Control & 0.78 & 0.46 & 177 & 125 & $83-330$ & \multirow[b]{2}{*}{ NS } \\
\hline & Enriched & 0.96 & 0.88 & 169 & 83 & $55-282$ & \\
\hline \multirow[t]{2}{*}{ 3,4-DHPEA-EDA glucuronide } & Control & 0.24 & $0 \cdot 21$ & 58 & 59 & $26-127$ & \multirow{2}{*}{ NS } \\
\hline & Enriched & $0 \cdot 20$ & $0 \cdot 18$ & 54 & 49 & $26-111$ & \\
\hline \multirow[t]{2}{*}{ 3,4-DHPEA-EA derivative } & Control & 0.36 & $0 \cdot 27$ & 75 & 62 & $5-235$ & \multirow{2}{*}{ NS } \\
\hline & Enriched & 0.60 & 0.59 & 98 & 78 & $7-245$ & \\
\hline \multirow[t]{2}{*}{ 3,4-DHPEA-EA derivative glucuronide } & Control & 0.04 & 0.03 & 6 & 6 & $2-14$ & \multirow[b]{2}{*}{ NS } \\
\hline & Enriched & 0.22 & 0.34 & 34 & 54 & $3-96$ & \\
\hline \multirow[t]{2}{*}{ Tyrosol sulphate } & Control & $1 \cdot 16$ & 1.07 & 228 & 189 & $59-601$ & \multirow[b]{2}{*}{ NS } \\
\hline & Enriched & 0.95 & 0.64 & 240 & 169 & $73-538$ & \\
\hline \multirow[t]{2}{*}{ 4-Methoxy-phenylacetic acid sulphate } & Control & 3.63 & $2 \cdot 20$ & 840 & 482 & $249-1891$ & \multirow[b]{2}{*}{ NS } \\
\hline & Enriched & $2 \cdot 38$ & 2.05 & 565 & 519 & $46-2024$ & \\
\hline \multirow[t]{2}{*}{ 4-Methoxy-phenylethanol sulphate } & Control & $2 \cdot 85$ & 1.74 & 617 & 378 & $119-1256$ & \multirow[b]{2}{*}{ NS } \\
\hline & Enriched & 2.44 & 2.59 & 611 & 645 & $106-2054$ & \\
\hline \multirow[t]{2}{*}{ 4-Methoxy-phenylethanol glucuronide } & Control & 0.03 & 0.01 & 6 & 5 & $2-9$ & \multirow{2}{*}{ NS } \\
\hline & Enriched & 0.03 & 0.01 & 6 & 5 & $3-9$ & \\
\hline
\end{tabular}

$C_{\max }$, maximum concentration of the metabolites; AUC, area under the curve; 3,4-DHPEA-EDA, dialdehydic form of elenolic acid linked to hydroxytyrosol; 3,4-DHPEA-EA, oleuropein aglycone.

Table 4. Pharmacokinetic parameters of the metabolites derived from the phenolic acids and the lignans in plasma samples after the intake of either control or phenol-enriched olive oil ( $n 13)$

(Mean values, standard deviations and ranges)

\begin{tabular}{|c|c|c|c|c|c|c|c|}
\hline \multirow[b]{2}{*}{ Phenolic compound } & \multirow[b]{2}{*}{ Oil } & \multicolumn{2}{|c|}{$C_{\max }(\mu \mathrm{mol} / \mathrm{l})$} & \multicolumn{2}{|c|}{$\begin{array}{c}\text { AUC } \\
(\mu \mathrm{mol} \times \min / l)\end{array}$} & \multirow{2}{*}{$\begin{array}{c}\text { AUC range } \\
(\mu \mathrm{mol} \times \mathrm{min} / \mathrm{l})\end{array}$} & \multirow[b]{2}{*}{$P$} \\
\hline & & Mean & SD & Mean & SD & & \\
\hline \multirow[t]{2}{*}{ Apigenin glucuronide } & Control & 0.09 & 0.05 & 16 & 7 & $8-35$ & \multirow{2}{*}{ NS } \\
\hline & Enriched & 0.08 & 0.03 & 16 & 9 & $8-36$ & \\
\hline \multirow[t]{2}{*}{ Hydroxybenzoic acid } & Control & 2.23 & 1.65 & 439 & 285 & $128-933$ & \multirow{2}{*}{ NS } \\
\hline & Enriched & 2.42 & $1 \cdot 71$ & 471 & 257 & $72-923$ & \\
\hline \multirow{2}{*}{ Hydroxyphenylacetic acid } & Control & 4.95 & 2.63 & 1,135 & 723 & $185-2454$ & \multirow[b]{2}{*}{ NS } \\
\hline & Enriched & $5 \cdot 79$ & $2 \cdot 67$ & 1,317 & 742 & $172-2507$ & \\
\hline \multirow[t]{2}{*}{ Vanillic acid } & Control & $6 \cdot 70$ & $2 \cdot 22$ & 1,254 & 512 & $824-1821$ & \multirow[b]{2}{*}{ NS } \\
\hline & Enriched & 3.19 & 0.45 & 538 & 166 & $368-700$ & \\
\hline \multirow[t]{2}{*}{ Vanillic acid sulphate } & Control & 0.64 & 0.75 & 137 & 161 & $32-416$ & \multirow{2}{*}{ NS } \\
\hline & Enriched & 0.75 & 0.39 & 174 & 87 & $75-232$ & \\
\hline \multirow[t]{2}{*}{ Caffeic acid } & Control & 4.06 & $2 \cdot 19$ & 798 & 530 & $284-1885$ & \multirow{2}{*}{ NS } \\
\hline & Enriched & 4.98 & 3.09 & 959 & 621 & $355-2094$ & \\
\hline \multirow[t]{2}{*}{ Caffeic acid sulphate } & Control & 1.20 & 0.87 & 226 & 181 & $19-477$ & \multirow[t]{2}{*}{ NS } \\
\hline & Enriched & 2.58 & 4.98 & 357 & 583 & $32-1818$ & \\
\hline \multirow[t]{2}{*}{ Coumaric acid sulphate } & Control & $1 \cdot 10$ & 0.60 & 222 & 129 & $63-533$ & \multirow[b]{2}{*}{ NS } \\
\hline & Enriched & $1 \cdot 19$ & 0.88 & 271 & 225 & $89-750$ & \\
\hline \multirow[t]{2}{*}{ Vanillin } & Control & 1.42 & 1.19 & 126 & 113 & $19-245$ & \multirow{2}{*}{ NS } \\
\hline & Enriched & 2.42 & 3.41 & 302 & 475 & $42-1014$ & \\
\hline \multirow[t]{2}{*}{ Vanillin sulphate } & Control & 12.96 & $10 \cdot 78$ & 2693 & 1970 & $718-6563$ & \multirow{2}{*}{$<0.05$} \\
\hline & Enriched & 23.55 & $11 \cdot 73$ & 5161 & 2852 & $1711-10800$ & \\
\hline \multirow[t]{2}{*}{ Vanillin glucuronide } & Control & 0.59 & 0.71 & 140 & 161 & $46-327$ & \multirow{2}{*}{ NS } \\
\hline & Enriched & 0.36 & 0.39 & 81 & 79 & $31-172$ & \\
\hline \multirow[t]{2}{*}{ Ferulic acid derivative } & Control & 0.27 & 0.03 & 70 & 6 & $49-131$ & \multirow{2}{*}{ NS } \\
\hline & Enriched & 0.23 & 0.05 & 51 & 12 & $39-71$ & \\
\hline Ferulic acid derivative sulphate & Control & 5.56 & $6 \cdot 54$ & 919 & 901 & $154-3245$ & \\
\hline & Enriched & $6 \cdot 74$ & 2.90 & 1,257 & 687 & $454-2575$ & NS \\
\hline Ferulic acid glucuronide & Control & 0.53 & 0.51 & 119 & 106 & $19-214$ & \\
\hline & Enriched & 0.77 & 0.73 & 189 & 166 & $23-413$ & NS \\
\hline
\end{tabular}

$C_{\text {max }}$, maximum concentration of the metabolites; AUC, area under the curve. 
Regarding the metabolites originating from phenolic acids, it could be considered that the compounds that appeared in the basal conditions are fermentation products, generated through the effect of the human gut microbiota on the dietary fibre and some phenolic compounds, such as flavonoids, in the habitual diet of the volunteers ${ }^{(30)}$. Once the VOO has been ingested, the increase in the concentration of the phenolic acid metabolites in the plasma over time could be partly attributed to the absorption of this fraction included in the olive oil. However, the enrichment of the olive oil with the phenolic extract also increases the concentration of the flavonoid group, and it is possible that this could generate a further increase in the concentration of the phenolic acid metabolites once the human gut microbiota act on them. Therefore, in addition to the direct effect on the content of the phenolic acid metabolites observed in the plasma during the period of study, it is possible that an indirect increase occurs through the fermentation of the flavonoids in the colon. Nevertheless, further studies would be needed to confirm this.

It is also remarkable that a certain shift in the time when the maximum concentration of some of the identified metabolites was reached was observed as a consequence of the phenol enrichment of the oil. As an example, Fig. 4 shows the graphical evaluation of the concentration of some metabolites from the different phenolic groups identified in the plasma after the intake of both the VOO and the EVOO: hydroxytyrosol sulphate (phenyl alcohol), oleuropein aglycone derivative glucuronide (secoiridoid), apigenin glucuronide (flavonoid) and ferulic acid glucuronide (phenolic acid). After the consumption of the VOO, the maximum concentration of these peaks was reached at $60 \mathrm{~min}$. However, consumption of the EVOO shifted this maximum to $120 \mathrm{~min}$. This could be due to a higher concentration leading to a certain saturation of

(a)

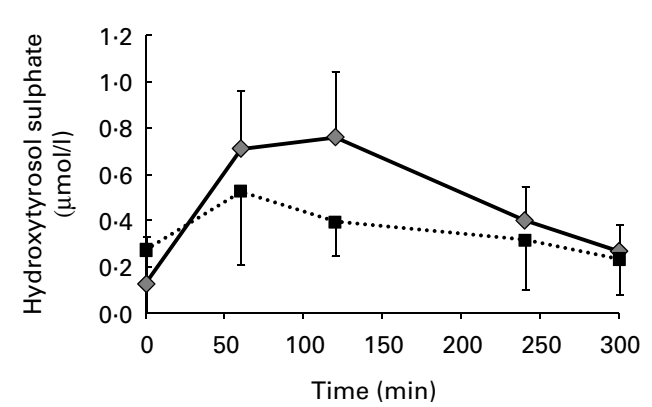

(c)

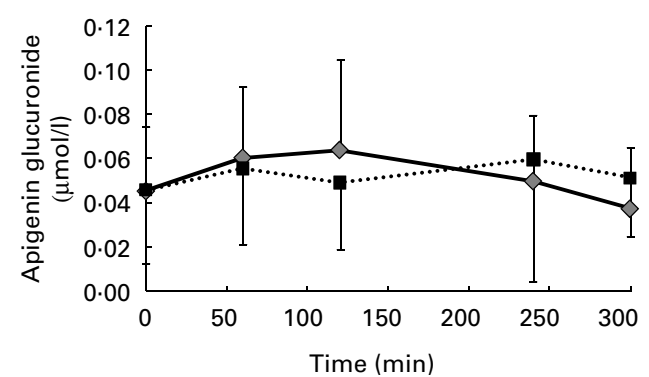

the mechanisms involved in the absorption and transport of these compounds in the human organism. Therefore, the phenol-enriched oil needs more time to reach the maximum concentration in the plasma.

In addition to this change in the time to reach the highest concentration, there was a general quantitative increment in the value of these metabolites in the plasma after the ingestion of the EVOO compared with the one obtained after consumption of the VOO. This is of great importance and points to the suitability of phenol enrichment of the oil as a means of increasing the amount of these desirable compounds in the organism, which is necessary to produce a real effect on the development of some types of cancers, such as colon cancer $^{(31)}$.

Analysing the pharmacokinetic parameters of the different groups of phenolic compounds under study, their different pattern of absorption could be evaluated. To start with, the time when the maximum concentration is reached suggests that their absorption occurs approximately in the small intestine after being subjected to the strong metabolism of the organism. Thus, hydroxytyrosol sulphate (and by extension the other phenyl alcohols metabolites) could come from the high spectra of secoiridoid derivatives in the oil by hydrolysis in the stomach (phase I metabolism) and later conjugation by the action of the sulphotransferases (phase II). As was reported in our previous study ${ }^{(28)}$, after simulated in vitro digestion of VOO, there was a significant increment in hydroxytyrosol in the stomach (rising by approximately $70 \%$ ) due to the acidic conditions of the media $(\mathrm{pH} 2)$. This can be related to the high proportion of phenyl alcohols conjugated as their different metabolites that appeared in the plasma. The absorption of phenolic acid metabolites seems to follow the same pattern.

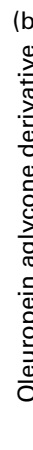

(d)

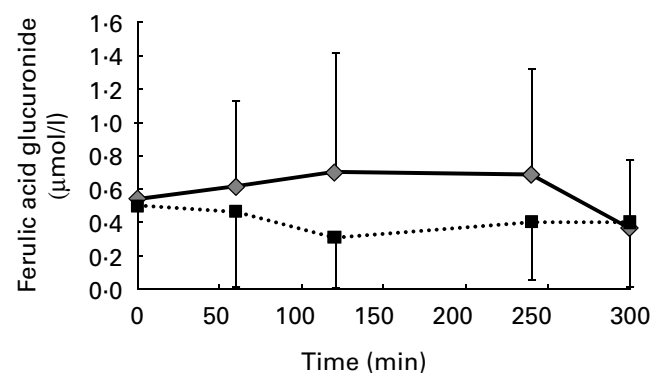

Fig. 4. Pharmacokinetics of (a) hydroxytyrosol sulphate, (b) oleuropein aglycone derivative glucuronide, (c) apigenin glucuronide and (d) ferulic acid glucuronide in human plasma after the intake of control $(\boldsymbol{\square})$ and phenol-enriched olive oil $(\diamond)$. Data are expressed as $\mu$ mol phenolic metabolite per litre plasma. 
On the other hand, the appearance of oleuropein aglycone derivative and its glucuronide form seems to suggest that some of the secoiridoids in the oil are able to resist the strong conditions of the digestion process and reach the blood stream in a chemical form higher than hydroxytyrosol. This is of great importance due to the healthy effects that these compounds could exert ${ }^{(23)}$.

The non-identification of any metabolic form derived from lignans in the plasma could be due to their degradation by the colonic flora of the gut and further transformation into enterodiol and enterolactone. This process is believed to take place in the period of $8-10 \mathrm{~h}$ after ingestion ${ }^{(32)}$.

\section{Concluding remarks}

The enrichment of olive oil with an olive cake extract increased the amount of phenolic compounds in the resulting oil and had a direct influence on their bioavailability. The in vivo study showed that the concentration of fourteen of twenty-four compounds detected was higher in the plasma samples from the EVOO than after ingestion of VOO. Among these, two of them, hydroxytyrosol sulphate and vanillin sulphate, were statistically significant in attending their pharmacokinetic parameters, demonstrating the suitability of enrichment. In general, a displacement of the time to reach the maximum concentration is observed in the samples, which indicates that more time is needed to absorb the higher phenolic content. However, inter-individual variability in the concentration of the plasma phenol metabolites shows that it is difficult to show statistically significant differences between the VOO and the EVOO. This could be due to the high number of variables that interfere in the absorption of these compounds and suggests that the metabolism of phenols is affected first by the individual. These preliminary results suggest the need for a major interventional study with a larger number of volunteers to confirm the effectiveness of the enrichment of olive oil in increasing the plasma concentration of the phenol metabolites.

\section{Acknowledgements}

The present study was supported by the Spanish Ministry of Education and Science financing the projects AGL200507881-C02-01/ALI and AGL2005-07881-C02-02/ALI; Health Ministry (FIS; PI021307) and CIBERDEM; and the grant received by Manuel Suarez (BES-2006-14136). We wish to thank the company Moli dels Torm S.L. (Els Torms, Lleida, Catalonia, Spain) for the supply of the olive cake samples to obtain phenol extract. The authors declare that there is no conflict of interest. M.-J. M. and R. S. had full access to all of the data in the study, and took responsibility for the integrity of the data and the accuracy of data analysis. M.-J. M. and R. S conceived the study concept and design. M. S., A. M. and M.-P. R. prepared and analysed the phenol-enriched olive oil. R. M. V., S. F., M. G. and R. S. conducted the human study. M. S. and A. M. had a role in the acquisition of in vivo bioavailability data. M. S., R. M. V., M.-J. M., R. S. and M. G. analysed and interpreted the data. M. S., M.-J. M.,
R. S. and R. M. V. drafted the manuscript. M.-J. M. and R. S. participated in the critical revision of the manuscript for important intellectual content. M. G. and S. F. provided administrative, technical and logistic support.

\section{References}

1. Knoops KTB, De Groot LCPGM, Kromhout D, et al. (2004) Mediterranean diet, lifestyle factors, and 10-year mortality in elderly European men and women: the HALE project. JAMA 292, 1433-1439.

2. Covas MI (2007) Olive oil and cardiovascular system. Pharmacol Res 55, 175-186.

3. Covas MI (2008) Bioactive effects of olive oil phenolic compounds in humans: reduction of heart disease factors and oxidative damage. Inflammopharmacology 16, 216-218.

4. Obied HK, Bedgood DR Jr., Prenzler PD, et al. (2007) Bioscreening of Australian olive mill waste extracts: biophenol content, antioxidant, antimicrobial and molluscicidal activities. Food Chem Toxicol 45, 1238-1248.

5. López-Miranda J, Pérez-Jiménez F, Ros E, et al. (2010) Olive oil and health: summary of the II international conference on olive oil and health consensus report, Jaén and Córdoba (Spain) 2008. Nutr Metab Cardiovasc Dis 20, 284-294.

6. Visioli F \& Galli C (2001) Antiatherogenic components of olive oil. Curr Atheroscler Rep 3, 64-67.

7. Owen RW, Giacosa A, Hull WE, et al. (2000) Olive-oil consumption and health: the possible role of antioxidants. Lancet Oncol 1, 107-112.

8. Manach C, Scalbert A, Morand C, et al. (2004) Polyphenols: food sources and bioavailability. Am J Clin Nutr 79, $727-747$.

9. Visioli F, Galli C, Bornet F, et al. (2000) Olive oil phenolics are dose-dependently absorbed in humans. FEBS Lett $\mathbf{4 6 8}$, 159-160.

10. Caruso D, Visioli F, Patelli R, et al. (2001) Urinary excretion of olive oil phenols and their metabolites in humans. Metabolism 50, 1426-1428.

11. Miró-Casas E, Covas M-I, Farre M, et al. (2003) Hydroxytyrosol disposition in humans. Clin Chem 49, 945-952.

12. Marrugat J, Covas MI, Fito M, et al. (2004) Effects of differing phenolic content in dietary olive oils on lipids and LDL oxidation - a randomized controlled trial. Eur J Nutr $\mathbf{4 3}$, $140-147$.

13. González-Santiago M, Fonollá J \& López-Huertas E (2010) Human absorption of a supplement containing purified hydroxytyrosol, a natural antioxidant from olive oil, and evidence for its transient association with low-density lipoproteins. Pharmacol Res 61, 364-370.

14. Paiva-Martins F, Fernandes J, Santos V, et al. (2010) Powerful protective role of 3,4-dihidroxyphenylethanol-elenolic acid dialdehyde against erythrocyte oxidative-induced hemolysis. J Agric Food Chem 58, 135-140.

15. Konstantinidou V, Covas M-I, Muñoz-Aguayo D, et al. (2010) In vivo nutrigenomics effects of virgin olive oil polyphenols within the frame of the Mediterranean diet: a randomized controlled trial. FASEB J 24, 2546-2557.

16. Vissers MN, Zock PL, Roodenburg AJ, et al. (2002) Olive oil phenols are absorbed in humans. J Nutr 132, 409-417.

17. Leenen R, Roodenburg AJ, Vissers MN, et al. (2002) Supplementation of plasma with olive oil phenols and extracts: influence on LDL oxidation. I Agric Food Chem 50, 1290-1297. 
18. Esti M, Cinquanta L \& La Notte E (1998) Phenolic compounds in different olive varieties. J Agric Food Chem 46, $32-35$.

19. Artajo LS, Romero MP, Morelloç JR, et al. (2006) Enrichment of refined olive oil with phenolic compounds: evaluation of their antioxidant activity and their effect on the bitter index. J Agric Food Chem 54, 6079-6088.

20. Suárez M, Romero MP, Ramo T, et al. (2009) Methods for preparing phenolic extracts from olive cake for potential application as food antioxidants. J Agric Food Chem 57, 1463-1472.

21. Suárez M, Macià A, Romero M-P, et al. (2008) Improved liquid chromatography tandem mass spectrometry method for the determination of phenolic compounds in virgin olive oil. J Chromatogr A 1214, 90-99.

22. Suárez M, Romero M-P, Macià A, et al. (2009) Improved method for identifying and quantifying olive oil phenolic compounds and their metabolites in human plasma by microelution solid-phase extraction plate and liquid chromatography-tandem mass spectrometry. J Chromatogr B $\mathbf{8 7 7}$, 4097-4106.

23. Paiva-Martins F, Fernandes J, Rocha S, et al. (2009) Effects of olive oil polyphenols on erythrocyte oxidative damage. Mol Nutr Food Res 53, 609-616.

24. De la Torre-Carbot K, Jauregui O, Castellote AI, et al. (2006) Rapid high-performance liquid chromatographyelectrospray ionization tandem mass spectrometry method for qualitative and quantitative analysis of virgin olive oil phenolic metabolites in human low-density lipoproteins. J Chromatogr A 1116, 69-75.

25. D'Angelo S, Manna C, Migliardi V, et al. (2001) Pharmacokinetics and metabolism of hydroxytyrosol, a natural antioxidant from olive oil. Drug Metab Dispos 29, 1492-1498.

26. García-Villalba R, Carrasco-Pancorbo A, Nevedomskaya E, et al. (2010) Exploratory analysis of human urine by LC-ESI-TOF MS after high intake of olive oil: understanding the metabolism of polyphenols. Anal Bioanal Chem $\mathbf{3 9 8}$, 463-475.

27. D'Archivio M, Filesi C, Vari R, et al. (2010) Bioavailability of polyphenols: status and controversies. Int J Mol Sci $\mathbf{1 1}$ $1321-1342$.

28. Suárez M, Romero M-P \& Motilva M-J (2010) Development of a phenol-enriched olive oil with phenolic compounds from olive cake. J Agric Food Chem 58, 10396-10403.

29. Soler A, Romero M-P, Macià A, et al. (2010) Digestion stability and evaluation of the metabolism and transport of olive oil phenols in the human small-intestinal epithelial Caco-2/TC7 cell line. Food Chem 119, 703-714.

30. Aura A-M (2008) Microbial metabolism of dietary phenolic compounds in the colon. Phytochem Rev 7, 407-429.

31. Colomer R \& Menéndez JA (2006) Mediterranean diet, olive oil and cancer. Clin Transl Oncol 8, 15-21.

32. Kuijsten A, Arts ICW, Vree TB, et al. (2005) Pharmacokinetics of enterolignans in healthy men and women consuming a single dose of secoisolariciresinol diglucoside. J Nutr $\mathbf{1 3 5}$, 795-801. 